\title{
On fractional differential inclusions with anti-periodic type integral boundary conditions
}

\author{
Bashir Ahmad', Sotiris K Ntouyas² and Ahmed Alsaedi ${ }^{*}$
}

"Correspondence:

aalsaedi@hotmail.com

'Department of Mathematics,

Faculty of Science, King Abdulaziz

University, P.O. Box 80203, Jeddah,

21589, Saudi Arabia

Full list of author information is

available at the end of the article

\begin{abstract}
This paper investigates the existence of solutions for fractional differential inclusions of order $q \in(2,3]$ with anti-periodic type integral boundary conditions by means of some standard fixed point theorems for inclusions. Our results include the cases when the multivalued map involved in the problem has convex as well as non-convex values. The paper concludes with an illustrative example.
\end{abstract}

MSC: $34 \mathrm{~A} 60 ; 34 \mathrm{~A} 08$

Keywords: fractional differential inclusions; anti-periodic; integral boundary conditions; fixed point theorems

\section{Introduction}

The topic of fractional differential equations and inclusions has recently emerged as a popular field of research due to its extensive development and applications in several disciplines such as physics, mechanics, chemistry, engineering, etc. [1-5]. An important characteristic of a fractional-order differential operator, in contrast to its integer-order counterpart, is its nonlocal nature. This feature of fractional-order operators (equations) is regarded as one of the key factors for the popularity of the subject. As a matter of fact, the use of fractional-order operators in the mathematical modeling of several real world processes gives rise to more realistic models as these operators are capable of describing memory and hereditary properties. For some recent results on fractional differential equations, see [6-22] and the references cited therein, whereas some recent work dealing with fractional differential inclusions can be found in [23-28].

In this paper, we study a boundary value problem of fractional differential inclusions with anti-periodic type integral boundary conditions given by

$$
\left\{\begin{array}{l}
{ }^{c} D^{q} x(t) x(t) \in F(t, x(t)), \quad 0<t<T, 2<q \leq 3, \\
x^{(j)}(0)-\lambda_{j} x^{(j)}(T)=\mu_{j} \int_{0}^{T} g_{j}(s, x(s)) d s, \quad j=0,1,2,
\end{array}\right.
$$

where ${ }^{c} D^{q}$ denotes the Caputo derivative of fractional order $q, x^{(j)}(\cdot)$ denotes $j$ th derivative of $x(\cdot)$ with $x^{(0)}(\cdot)=x(\cdot), F:[0, T] \times \mathbb{R} \rightarrow \mathcal{P}(\mathbb{R})$ is a multivalued map, $\mathcal{P}(\mathbb{R})$ is the family of all subsets of $\mathbb{R}, g_{j}:[0, T] \times \mathbb{R} \rightarrow \mathbb{R}$ are given continuous functions and $\lambda_{j}, \mu_{j} \in \mathbb{R}\left(\lambda_{j} \neq 1\right)$.

The present work is motivated by a recent paper [22], where the authors considered (1.1) with $F$ as a single-valued map. The existence of solutions for problem (1.1) has been dis- 
cussed for the cases when the right-hand side is convex as well as non-convex valued. The first result is based on the nonlinear alternative of Leray-Schauder type, whereas the second result is established by combining the nonlinear alternative of Leray-Schauder type for single-valued maps with a selection theorem due to Bressan and Colombo for lower semicontinuous multivalued maps with nonempty closed and decomposable values. In the third result, we use the fixed point theorem for contraction multivalued maps due to Covitz and Nadler. Though the methods used are well known, their exposition in the framework of problem (1.1) is new. We recall some preliminary facts about fractional calculus and multivalued maps in Section 2, while the main results are presented in Section 3.

\section{Preliminaries}

\subsection{Fractional calculus}

Let us recall some basic definitions of fractional calculus [1-3].

Definition 2.1 Let $h:[0, \infty) \rightarrow \mathbb{R}$ be an $(n-1)$-times absolutely continuous function. Then the Caputo derivative of fractional order $v$ for $h$ is defined as

$$
{ }^{c} D^{v} h(t)=\frac{1}{\Gamma(n-v)} \int_{0}^{t}(t-s)^{n-v-1} h^{(n)}(s) d s, \quad n-1<v<n, n=[v]+1,
$$

where $[v]$ denotes the integer part of the real number $v$.

Definition 2.2 The Riemann-Liouville fractional integral of order $v$ is defined as

$$
I^{v} h(t)=\frac{1}{\Gamma(v)} \int_{0}^{t} \frac{g(s)}{(t-s)^{1-v}} d s, \quad v>0
$$

provided the integral exists.

Definition 2.3 A function $x \in A C^{1}([0, T], \mathbb{R})$ is called a solution of problem (1.1) if there exists a function $v \in L^{1}([0, T], \mathbb{R})$ with $v(t) \in F(t, x(t))$, a.e. $[0, T]$ such that $D^{\alpha} x(t)=v(t)$, a.e. $[0,1]$ and $x^{(j)}(0)-\lambda_{j} x^{(j)}(T)=\mu_{j} \int_{0}^{T} g_{j}(s, x(s)) d s, j=0,1,2$.

In the sequel, the following lemma plays a pivotal role.

Lemma 2.4 ([22]) For a given $y \in C([0, T], \mathbb{R})$ and $2<q \leq 3$, the unique solution of the equation ${ }^{c} D^{q} x(t)=y(t), t \in[0, T]$ subject to the boundary conditions of (1.1) is given by

$$
\begin{aligned}
x(t)= & \int_{0}^{t} \frac{(t-s)^{q-1}}{\Gamma(q)} y(s) d s-\lambda_{0} \xi_{1} \int_{0}^{T} \frac{(T-s)^{q-1}}{\Gamma(q)} y(s) d s \\
& +\lambda_{1} \eta_{2} \int_{0}^{T} \frac{(T-s)^{q-2}}{\Gamma(q-1)} y(s) d s+\lambda_{2} \eta_{1} \int_{0}^{T} \frac{(T-s)^{q-3}}{\Gamma(q-2)} y(s) d s \\
& -\mu_{0} \xi_{1} \int_{0}^{T} g_{0}(s, x(s)) d s+\mu_{1} \eta_{2} \int_{0}^{T} g_{1}(s, x(s)) d s \\
& +\mu_{2} \eta_{1} \int_{0}^{T} g_{2}(s, x(s)) d s,
\end{aligned}
$$


where

$$
\begin{aligned}
& \eta_{1}=\xi_{3}\left[-\lambda_{0}\left(\lambda_{1}+1\right) T^{2}+2 \lambda_{1}\left(\lambda_{0}-1\right) t T-\left(\lambda_{0}-1\right)\left(\lambda_{1}-1\right) t^{2}\right], \\
& \eta_{2}=\xi_{2}\left[\lambda_{0} T-\left(\lambda_{0}-1\right) t\right], \\
& \xi_{1}=\frac{1}{\lambda_{0}-1}, \quad \xi_{2}=\frac{1}{\left(\lambda_{0}-1\right)\left(\lambda_{1}-1\right)}, \quad \xi_{3}=\frac{1}{2\left(\lambda_{0}-1\right)\left(\lambda_{1}-1\right)\left(\lambda_{2}-1\right)} .
\end{aligned}
$$

\subsection{Basic concepts of multivalued analysis}

Let us begin this section with some basic concepts of multi-valued maps $[29,30]$.

Let $\mathcal{X}$ denote a normed space equipped with the norm $|\cdot|$. A multivalued map $\mathcal{G}: \mathcal{X} \rightarrow$ $\mathcal{P}(\mathcal{X})$ is

- convex (closed) valued if $\mathcal{G}(x)$ is convex (closed) for all $x \in \mathcal{X}$;

- bounded on bounded sets if $\mathcal{G}(B)=\bigcup_{x \in B} \mathcal{G}(x)$ is bounded in $\mathcal{X}$ for all bounded sets $B$ in $\mathcal{X}$, that is, $\sup _{x \in B}\{\sup \{|y|: y \in \mathcal{G}(x)\}\}<\infty$;

- upper semi-continuous (u.s.c.) on $\mathcal{X}$ if for each $x_{0} \in \mathcal{X}$, the set $\mathcal{G}\left(x_{0}\right)$ is a nonempty closed subset of $\mathcal{X}$, and if for each open set $\mathcal{N}$ of $\mathcal{X}$ containing $\mathcal{G}\left(x_{0}\right)$, there exists an open neighborhood $\mathcal{N}_{0}$ of $x_{0}$ such that $\mathcal{G}\left(\mathcal{N}_{0}\right) \subseteq \mathcal{N}$;

- completely continuous if $\mathcal{G}(B)$ is relatively compact for every bounded set $B$ in $\mathcal{X}$.

Remark 2.5 If the multivalued map $\mathcal{G}$ is completely continuous with nonempty compact values, then $\mathcal{G}$ is u.s.c. if and only if $\mathcal{G}$ has a closed graph, that is, $x_{n} \longrightarrow x_{*}, y_{n} \longrightarrow y_{*}$, $y_{n} \in \mathcal{G}\left(x_{n}\right)$ imply that $y_{*} \in G\left(x_{*}\right)$.

Definition 2.6 The multivalued map $\mathcal{G}$ has a fixed point if there is $x \in \mathcal{X}$ such that $x \in$ $\mathcal{G}(x)$. The fixed point set of the map $\mathcal{G}$ is denoted by Fix $G$.

Definition 2.7 A multivalued map $\mathcal{G}:[0, T] \rightarrow \mathcal{P}(\mathbb{R})$ with nonempty compact convex values is said to be measurable if for any $x \in \mathbb{R}$, the function

$$
t \longmapsto d(x, F(t))=\inf \{|x-y|: y \in F(t)\}
$$

is measurable.

Let $C([0, T], \mathbb{R})$ denote the Banach space of all continuous functions from $[0, T]$ into $\mathbb{R}$ with the norm

$$
\|x\|_{\infty}=\sup \{|x(t)|: t \in[0, T]\} .
$$

Let $L^{1}([0, T], \mathbb{R})$ be the Banach space of measurable functions $x:[0, T] \longrightarrow \mathbb{R}$ which are Lebesgue integrable and normed by

$$
\|x\|_{L^{1}}=\int_{0}^{T}|x(t)| d t \quad \text { for all } x \in L^{1}([0, T], \mathbb{R}) .
$$

Definition 2.8 A multivalued map $\mathcal{G}:[0, T] \times \mathbb{R} \rightarrow \mathcal{P}(\mathbb{R})$ is called Carathéodory if $t \longmapsto$ $\mathcal{G}(t, x)$ is measurable for each $x \in \mathbb{R}$ and $x \longmapsto \mathcal{G}(t, x)$ is upper semicontinuous for almost all $t \in[0, T]$. A Carathéodory function $\mathcal{G}$ is said to be $L^{1}$-Carathéodory if, for each $\delta>0$, there 
exists $\varphi_{\delta} \in L^{1}\left([0, T], \mathbb{R}^{+}\right)$such that $\|\mathcal{G}(t, x)\|=\sup \{|v|: v \in \mathcal{G}(t, x)\} \leq \varphi_{\delta}(t)$ for all $\|x\|_{\infty} \leq \delta$ and for a.e. $t \in[0, T]$.

For each $y \in C([0, T], \mathbb{R})$, we define the set of selections of $F$ by

$$
S_{F, y}:=\left\{v \in L^{1}([0, T], \mathbb{R}): v(t) \in F(t, y(t)) \text { for a.e. } t \in[0, T]\right\} .
$$

Let $\mathcal{W}$ denote a nonempty closed subset of a Banach space $E$, and let $\mathcal{G}: \mathcal{W} \rightarrow \mathcal{P}(E)$ be a multivalued operator with nonempty closed values. The map $G$ is lower semi-continuous (l.s.c.) if the set $\{y \in \mathcal{W}: G(y) \cap B \neq \emptyset\}$ is open for any open set $B$ in $E$. Let $A$ be a subset of $[0, T] \times \mathbb{R} . A$ is $\mathcal{L} \otimes \mathcal{B}$ measurable if $A$ belongs to the $\sigma$-algebra generated by all sets of the form $\mathcal{J} \times \mathcal{D}$, where $\mathcal{J}$ is Lebesgue measurable in $[0, T]$ and $\mathcal{D}$ is Borel measurable in $\mathbb{R}$. A subset $\mathcal{A}$ of $L^{1}([0, T], \mathbb{R})$ is decomposable if for all $u, v \in \mathcal{A}$ and measurable $\mathcal{J} \subset[0, T]=$ $J$, the function $u \chi_{\mathcal{J}}+v \chi_{\mathcal{J}-\mathcal{J}} \in \mathcal{A}$, where $\chi_{\mathcal{J}}$ stands for the characteristic function of $\mathcal{J}$.

Definition 2.9 Let $Y$ be a separable metric space. A multivalued operator $N: Y \rightarrow$ $\mathcal{P}\left(L^{1}([0, T], \mathbb{R})\right)$ has the property $(\mathrm{BC})$ if $N$ is lower semi-continuous (l.s.c.) and has nonempty closed and decomposable values.

Let $F:[0, T] \times \mathbb{R} \rightarrow \mathcal{P}(\mathbb{R})$ be a multivalued map with nonempty compact values. Define a multivalued operator $\mathcal{F}: C([0, T] \times \mathbb{R}) \rightarrow \mathcal{P}\left(L^{1}([0, T], \mathbb{R})\right)$ associated with $F$ as

$$
\mathcal{F}(x)=\left\{w \in L^{1}([0, T], \mathbb{R}): w(t) \in F(t, x(t)) \text { for a.e. } t \in[0,1]\right\},
$$

which is called the Nemytskii operator associated with $F$.

Definition 2.10 Let $F:[0, T] \times \mathbb{R} \rightarrow \mathcal{P}(\mathbb{R})$ be a multivalued function with nonempty compact values. We say $F$ is of lower semi-continuous type (l.s.c. type) if its associated Nemytskii operator $\mathcal{F}$ is lower semi-continuous and has nonempty closed and decomposable values.

Let $(\mathcal{X}, d)$ be a metric space induced from the normed space $(\mathcal{X} ;\|\cdot\|)$. Consider $H_{d}$ : $\mathcal{P}(\mathcal{X}) \times \mathcal{P}(\mathcal{X}) \rightarrow \mathbb{R} \cup\{\infty\}$ given by

$$
H_{d}(A, B)=\max \left\{\sup _{a \in A} d(a, B), \sup _{b \in B} d(A, b)\right\},
$$

where $d(A, b)=\inf _{a \in A} d(a ; b)$ and $d(a, B)=\inf _{b \in B} d(a ; b)$. Then $\left(\mathcal{P}_{b, c l}(\mathcal{X}), H_{d}\right)$ is a metric space and $\left(\mathcal{P}_{c l}(\mathcal{X}), H_{d}\right)$ is a generalized metric space (see [31]), where $\mathcal{P}_{c l}(\mathcal{X})=\{Y \in \mathcal{P}(\mathcal{X})$ : $Y$ is closed $\}$, and $\mathcal{P}_{b, c l}(\mathcal{X})=\{Y \in \mathcal{P}(\mathcal{X}): Y$ is bounded and closed $\}$.

Definition 2.11 A multivalued operator $N: \mathcal{X} \rightarrow \mathcal{P}_{c l}(\mathcal{X})$ is called $\gamma$-Lipschitz if and only if there exists $\gamma>0$ such that $H_{d}(N(x), N(y)) \leq \gamma d(x, y)$ for each $x, y \in(\mathcal{X})$ and is a contraction if and only if it is $\gamma$-Lipschitz with $\gamma<1$.

\section{Existence results}

\subsection{The Carathéodory case}

We recall the following lemmas to prove the existence of solutions for problem (1.1) when the multivalued map $F$ in (1.1) is of Carathéodory type. 
Lemma 3.1 (Nonlinear alternative for Kakutani maps) [32] Let E be a Banach space, let $C$ be a closed convex subset of $E$, let $U$ be an open subset of $C$, and $0 \in U$. Suppose that $F: \bar{U} \rightarrow \mathcal{P}_{c p, c}(C)$ is an upper semicontinuous compact map; here $\mathcal{P}_{c p, c}(C)$ denotes the family of nonempty, compact convex subsets of $C$. Then either

(i) F has a fixed point in $\bar{U}$, or

(ii) there is an $u \in \partial U$ and $\lambda \in(0,1)$ with $u \in \lambda F(u)$.

Lemma 3.2 ([33]) Let $\mathcal{X}$ be a Banach space, and let $\mathcal{P}_{c p, c}(\mathcal{X})$ denote a family of nonempty, compact and convex subsets of $\mathcal{X}$. Let $F:[0, T] \times \mathbb{R} \rightarrow \mathcal{P}_{c p, c}(X)$ be an $L^{1}$-Carathéodory multivalued map, and let $\Theta$ be a linear continuous mapping from $L^{1}([0, T], \mathcal{X})$ to $C([0, T], \mathcal{X})$. Then the operator

$$
\Theta \circ S_{F}: C([0, T], \mathcal{X}) \rightarrow \mathcal{P}_{c p, c}(C([0, T], \mathcal{X})), \quad x \mapsto\left(\Theta \circ S_{F}\right)(x)=\Theta\left(S_{F, x}\right)
$$

is a closed graph operator in $C([0, T], \mathcal{X}) \times C([0, T], \mathcal{X})$.

Theorem 3.3 Suppose that

$\left(\mathrm{H}_{1}\right) \quad F:[0, T] \times \mathbb{R} \rightarrow \mathcal{P}(\mathbb{R})$ is Carathéodory and has nonempty compact and convex values;

$\left(\mathrm{H}_{2}\right)$ there exists a continuous nondecreasing function $\psi:[0, \infty) \rightarrow(0, \infty)$ and a function $p \in L^{1}\left([0, T], \mathbb{R}^{+}\right)$such that

$$
\|F(t, x)\|_{\mathcal{P}}:=\sup \{|y|: y \in F(t, x)\} \leq p(t) \psi(\|x\|) \quad \text { for each }(t, x) \in[0, T] \times \mathbb{R}
$$

$\left(\mathrm{H}_{3}\right)$ there exist continuous nondecreasing functions $\psi_{j}:[0, \infty) \rightarrow(0, \infty)$ and functions $p_{j} \in$ $L^{1}\left([0, T], \mathbb{R}^{+}\right)$such that

$$
\left|g_{j}(t, x)\right| \leq p_{j}(t) \psi_{j}(\|x\|), \quad j=0,1,2, \text { for each }(t, x) \in[0, T] \times \mathbb{R} ;
$$

$\left(\mathrm{H}_{4}\right)$ there exists a constant $M>0$ such that

$$
\frac{M}{\psi(M) \Omega_{1}\|p\|_{L^{1}}+\psi_{0}(M)\left|\mu_{0} \xi_{1}\right|\left\|p_{0}\right\|_{L^{1}}+\psi_{1}(M)\left|\mu_{1} \eta_{2}\right|\left\|p_{1}\right\|_{L^{1}}+\psi_{2}(M)\left|\mu_{2} \eta_{1}\right|\left\|p_{2}\right\|_{L^{1}}}>1,
$$

where

$$
\Omega_{1}=\frac{T^{q-1}}{\Gamma(q)}\left\{1+\left|\lambda_{0} \xi_{1}\right|+\left|\lambda_{1} \eta_{2}\right|(q-1) T^{-1}+\left|\lambda_{2} \eta_{1}\right| q(q-1)(q-2) T^{-2}\right\} .
$$

Then the boundary value problem (1.1) has at least one solution on $[0, T]$.

Proof Define the operator $\Omega_{F}: C([0, T], \mathbb{R}) \rightarrow \mathcal{P}(C([0, T], \mathbb{R}))$ by

$$
\Omega_{F}(x)=\left\{\begin{array}{l}
h \in C([0, T], \mathbb{R}): \\
h(t)=\left\{\begin{array}{l}
\int_{0}^{t} \frac{(t-s)^{q-1}}{\Gamma(q)} v(s) d s-\lambda_{0} \xi_{1} \int_{0}^{T} \frac{(T-s)^{q-1}}{\Gamma(q)} v(s) d s \\
+\lambda_{1} \eta_{2} \int_{0}^{T} \frac{(T-s)^{q-2}}{\Gamma(q-1)} v(s) d s+\lambda_{2} \eta_{1} \int_{0}^{T} \frac{(T-s)^{q-3}}{\Gamma(q-2)} v(s) d s \\
-\mu_{0} \xi_{1} \int_{0}^{T} g_{0}(s, x(s)) d s+\mu_{1} \eta_{2} \int_{0}^{T} g_{1}(s, x(s)) d s \\
+\mu_{2} \eta_{1} \int_{0}^{T} g_{2}(s, x(s)) d s, \quad 0 \leq t \leq 1
\end{array}\right\}
\end{array}\right.
$$


for $v \in S_{F, x}$. We will show that $\Omega_{F}$ satisfies the assumptions of the nonlinear alternative of Leray-Schauder type. The proof consists of several steps. As the first step, we show that $\Omega_{F}$ is convex for each $x \in C([0, T], \mathbb{R})$. This step is obvious since $S_{F, x}$ is convex ( $F$ has convex values), and therefore we omit the proof.

In the second step, we show that $\Omega_{F}$ maps bounded sets (balls) into bounded sets in $C([0, T], \mathbb{R})$. For a positive number $\rho$, let $B_{\rho}=\{x \in C([0, T], \mathbb{R}):\|x\| \leq \rho\}$ be a bounded ball in $C([0, T], \mathbb{R})$. Then, for each $h \in \Omega_{F}(x), x \in B_{\rho}$, there exists $v \in S_{F, x}$ such that

$$
\begin{aligned}
h(t)= & \int_{0}^{t} \frac{(t-s)^{q-1}}{\Gamma(q)} v(s) d s-\lambda_{0} \xi_{1} \int_{0}^{T} \frac{(T-s)^{q-1}}{\Gamma(q)} v(s) d s \\
& +\lambda_{1} \eta_{2} \int_{0}^{T} \frac{(T-s)^{q-2}}{\Gamma(q-1)} v(s) d s+\lambda_{2} \eta_{1} \int_{0}^{T} \frac{(T-s)^{q-3}}{\Gamma(q-2)} v(s) d s \\
& -\mu_{0} \xi_{1} \int_{0}^{T} g_{0}(s, x(s)) d s+\mu_{1} \eta_{2} \int_{0}^{T} g_{1}(s, x(s)) d s+\mu_{2} \eta_{1} \int_{0}^{T} g_{2}(s, x(s)) d s .
\end{aligned}
$$

Then for $t \in[0, T]$ we have

$$
\begin{aligned}
|h(t)| \leq & \int_{0}^{t} \frac{(t-s)^{q-1}}{\Gamma(q)}|v(s)| d s+\left|\lambda_{0} \xi_{1}\right| \int_{0}^{T} \frac{(T-s)^{q-1}}{\Gamma(q)}|v(s)| d s \\
& +\left|\lambda_{1} \eta_{2}\right| \int_{0}^{T} \frac{(T-s)^{q-2}}{\Gamma(q-1)}|v(s)| d s+\left|\lambda_{2} \eta_{1}\right| \int_{0}^{T} \frac{(T-s)^{q-3}}{\Gamma(q-2)}|v(s)| d s \\
& +\left|\mu_{0} \xi_{1}\right| \int_{0}^{T}\left|g_{0}(s, x(s))\right| d s+\left|\mu_{1} \eta_{2}\right| \int_{0}^{T}\left|g_{1}(s, x(s))\right| d s \\
& +\left|\mu_{2} \eta_{1}\right| \int_{0}^{T}\left|g_{2}(s, x(s))\right| d s \\
\leq & \psi(\|x\|)\left\{\frac{T^{q-1}}{\Gamma(q)}+\left|\lambda_{0} \xi_{1}\right| \frac{T^{q-1}}{\Gamma(q)}+\left|\lambda_{1} \eta_{2}\right| \frac{T^{q-2}}{\Gamma(q-1)}+\left|\lambda_{2} \eta_{1}\right| \frac{T^{q-3}}{\Gamma(q-2)}\right\} \int_{0}^{T} p(s) d s \\
& +\psi_{0}(\|x\|)\left|\mu_{0} \xi_{1}\right| \int_{0}^{T} p_{0}(s) d s+\psi_{1}(\|x\|)\left|\mu_{1} \eta_{2}\right| \int_{0}^{T} p_{1}(s) d s \\
& +\psi_{2}(\|x\|)\left|\mu_{2} \eta_{1}\right| \int_{0}^{T} p_{2}(s) d s \\
\leq & \psi(\|x\|) \Omega_{1}\|p\|_{L^{1}}+\psi_{0}(\|x\|)\left|\mu_{0} \xi_{1}\right|\left\|p_{0}\right\|_{L^{1}}+\psi_{1}(\|x\|)\left|\mu_{1} \eta_{2}\right|\left\|p_{1}\right\|_{L^{1}} \\
& +\psi_{2}(\|x\|)\left|\mu_{2} \eta_{1}\right|\left\|p_{2}\right\|_{L^{1}} .
\end{aligned}
$$

Thus,

$$
\|h\| \leq \psi(\rho) \Omega_{1}\|p\|_{L^{1}}+\psi_{0}(\rho)\left|\mu_{0} \xi_{1}\right|\left\|p_{0}\right\|_{L^{1}}+\psi_{1}(\rho)\left|\mu_{1} \eta_{2}\right|\left\|p_{1}\right\|_{L^{1}}+\psi_{2}(\rho)\left|\mu_{2} \eta_{1}\right|\left\|p_{2}\right\|_{L^{1}}
$$

Now we show that $\Omega_{F}$ maps bounded sets into equicontinuous sets of $C([0, T], \mathbb{R})$. Let $t^{\prime}, t^{\prime \prime} \in[0, T]$ with $t^{\prime}<t^{\prime \prime}$ and $x \in B_{\rho}$. For each $h \in \Omega_{F}(x)$, we obtain

$$
\begin{aligned}
& \left|(F x)\left(t^{\prime \prime}\right)-(F x)\left(t^{\prime}\right)\right| \\
& \quad \leq\left|\psi(\|x\|) \int_{0}^{t^{\prime}}\left[\frac{\left(t^{\prime \prime}-s\right)^{q-1}-\left(t^{\prime}-s\right)^{q-1}}{\Gamma(q)}\right] p(s) d s+\psi(\|x\|) \int_{t^{\prime}}^{t^{\prime \prime}} \frac{\left(t^{\prime \prime}-s\right)^{q-1}}{\Gamma(q)} p(s) d s\right|
\end{aligned}
$$




$$
\begin{aligned}
& +\left|\left(1-\lambda_{0}\right) \lambda_{1} \xi_{2}\right|\left|t^{\prime \prime}-t^{\prime}\right| \psi(\|x\|) \int_{0}^{T} \frac{(T-s)^{q-2}}{\Gamma(q-1)} p(s) d s \\
& +\left|\lambda_{2} \xi_{3}\right|\left[2\left|\left(1-\lambda_{0}\right) \lambda_{1}\right| T\left|t^{\prime \prime}-t^{\prime}\right|\right. \\
& \left.+\left|\left(1-\lambda_{0}\right)\left(1-\lambda_{1}\right)\right|\left|t^{\prime \prime 2}-t^{\prime 2}\right|\right] \psi(\|x\|) \int_{0}^{T} \frac{(T-s)^{q-3}}{\Gamma(q-2)} p(s) d s \\
& +\left|\left(1-\lambda_{0}\right) \mu_{1} \lambda_{1} \xi_{2}\right|\left|t^{\prime \prime}-t^{\prime}\right| \psi_{1}(\|x\|) \int_{0}^{T} p_{1}(s) d s \\
& +\left|\lambda_{2} \xi_{3} \mu_{2}\right|\left[2\left|\left(1-\lambda_{0}\right) \lambda_{1}\right| T\left|t^{\prime \prime}-t^{\prime}\right|\right. \\
& \left.+\left|\left(1-\lambda_{0}\right)\left(1-\lambda_{1}\right)\right|\left|t^{\prime \prime 2}-t^{\prime 2}\right|\right] \psi_{2}(\|x\|) \int_{0}^{T} p_{2}(s) d s .
\end{aligned}
$$

Obviously, the right-hand side of the above inequality tends to zero independently of $x \in$ $B_{\rho}$ as $t^{\prime \prime}-t^{\prime} \rightarrow 0$. As $\Omega_{F}$ satisfies the above three assumptions, it follows by the AscoliArzelá theorem that $\Omega_{F}: C([0, T], \mathbb{R}) \rightarrow \mathcal{P}(C([0, T], \mathbb{R}))$ is completely continuous.

In our next step, we show that $\Omega_{F}$ has a closed graph. Let $x_{n} \rightarrow x_{*}, h_{n} \in \Omega_{F}\left(x_{n}\right)$ and $h_{n} \rightarrow h_{*}$. Then we need to show that $h_{*} \in \Omega_{F}\left(x_{*}\right)$. Associated with $h_{n} \in \Omega_{F}\left(x_{n}\right)$, there exists $v_{n} \in S_{F, x_{n}}$ such that for each $t \in[0, T]$,

$$
\begin{aligned}
h_{n}(t)= & \int_{0}^{t} \frac{(t-s)^{q-1}}{\Gamma(q)} v_{n}(s) d s-\lambda_{0} \xi_{1} \int_{0}^{T} \frac{(T-s)^{q-1}}{\Gamma(q)} v_{n}(s) d s \\
& +\lambda_{1} \eta_{2} \int_{0}^{T} \frac{(T-s)^{q-2}}{\Gamma(q-1)} v_{n}(s) d s+\lambda_{2} \eta_{1} \int_{0}^{T} \frac{(T-s)^{q-3}}{\Gamma(q-2)} v_{n}(s) d s \\
& -\mu_{0} \xi_{1} \int_{0}^{T} g_{0}\left(s, x_{n}(s)\right) d s+\mu_{1} \eta_{2} \int_{0}^{T} g_{1}\left(s, x_{n}(s)\right) d s \\
& +\mu_{2} \eta_{1} \int_{0}^{T} g_{2}\left(s, x_{n}(s)\right) d s .
\end{aligned}
$$

Thus it suffices to show that there exists $v_{*} \in S_{F, x_{*}}$ such that for each $t \in[0, T]$,

$$
\begin{aligned}
h_{*}(t)= & \int_{0}^{t} \frac{(t-s)^{q-1}}{\Gamma(q)} v_{*}(s) d s-\lambda_{0} \xi_{1} \int_{0}^{T} \frac{(T-s)^{q-1}}{\Gamma(q)} v_{*}(s) d s \\
& +\lambda_{1} \eta_{2} \int_{0}^{T} \frac{(T-s)^{q-2}}{\Gamma(q-1)} v_{*}(s) d s+\lambda_{2} \eta_{1} \int_{0}^{T} \frac{(T-s)^{q-3}}{\Gamma(q-2)} v_{*}(s) d s \\
& -\mu_{0} \xi_{1} \int_{0}^{T} g_{0}\left(s, x_{*}(s)\right) d s+\mu_{1} \eta_{2} \int_{0}^{T} g_{1}\left(s, x_{*}(s)\right) d s+\mu_{2} \eta_{1} \int_{0}^{T} g_{2}\left(s, x_{*}(s)\right) d s .
\end{aligned}
$$

Let us consider the continuous linear operator $\Theta: L^{1}([0, T], \mathbb{R}) \rightarrow C([0, T], \mathbb{R})$ given by

$$
\begin{aligned}
f \mapsto \Theta(f)(t)= & \int_{0}^{t} \frac{(t-s)^{q-1}}{\Gamma(q)} v(s) d s-\lambda_{0} \xi_{1} \int_{0}^{T} \frac{(T-s)^{q-1}}{\Gamma(q)} v(s) d s \\
& +\lambda_{1} \eta_{2} \int_{0}^{T} \frac{(T-s)^{q-2}}{\Gamma(q-1)} v(s) d s+\lambda_{2} \eta_{1} \int_{0}^{T} \frac{(T-s)^{q-3}}{\Gamma(q-2)} v(s) d s \\
& -\mu_{0} \xi_{1} \int_{0}^{T} g_{0}(s, x(s)) d s+\mu_{1} \eta_{2} \int_{0}^{T} g_{1}(s, x(s)) d s \\
& +\mu_{2} \eta_{1} \int_{0}^{T} g_{2}(s, x(s)) d s .
\end{aligned}
$$


Observe that

$$
\begin{aligned}
& \left\|h_{n}(t)-h_{*}(t)\right\| \\
& \leq \int_{0}^{t} \frac{(t-s)^{q-1}}{\Gamma(q)}\left(v_{n}(s)-v_{*}(s)\right) d s-\lambda_{0} \xi_{1} \int_{0}^{T} \frac{(T-s)^{q-1}}{\Gamma(q)}\left(v_{n}(s)-v_{*}(s)\right) d s \\
& \quad+\lambda_{1} \eta_{2} \int_{0}^{T} \frac{(T-s)^{q-2}}{\Gamma(q-1)}\left(v_{n}(s)-v_{*}(s)\right) d s+\lambda_{2} \eta_{1} \int_{0}^{T} \frac{(T-s)^{q-3}}{\Gamma(q-2)}\left(v_{n}(s)-v_{*}(s)\right) d s \\
& \quad-\mu_{0} \xi_{1} \int_{0}^{T}\left(g_{0}\left(s, x_{n}(s)\right)-g_{0}\left(s, x_{*}(s)\right)\right) d s+\mu_{1} \eta_{2} \int_{0}^{T}\left(g_{1}\left(s, x_{n}(s)\right)-g_{1}\left(s, x_{*}(s)\right)\right) d s \\
& \quad+\mu_{2} \eta_{1} \int_{0}^{T}\left(g_{2}\left(s, x_{n}(s)\right)-g_{2}\left(s, x_{*}(s)\right)\right) d s .
\end{aligned}
$$

Thus, it follows by Lemma 3.2 that $\Theta \circ S_{F}$ is a closed graph operator. Further, we have $h_{n}(t) \in \Theta\left(S_{F, x_{n}}\right)$. Since $x_{n} \rightarrow x_{*}$, therefore, we have

$$
\begin{aligned}
h_{*}(t)= & \int_{0}^{t} \frac{(t-s)^{q-1}}{\Gamma(q)} v_{*}(s) d s-\lambda_{0} \xi_{1} \int_{0}^{T} \frac{(T-s)^{q-1}}{\Gamma(q)} v_{*}(s) d s \\
& +\lambda_{1} \eta_{2} \int_{0}^{T} \frac{(T-s)^{q-2}}{\Gamma(q-1)} v_{*}(s) d s+\lambda_{2} \eta_{1} \int_{0}^{T} \frac{(T-s)^{q-3}}{\Gamma(q-2)} v_{*}(s) d s \\
& -\mu_{0} \xi_{1} \int_{0}^{T} g_{0}\left(s, x_{*}(s)\right) d s+\mu_{1} \eta_{2} \int_{0}^{T} g_{1}\left(s, x_{*}(s)\right) d s \\
& +\mu_{2} \eta_{1} \int_{0}^{T} g_{2}\left(s, x_{*}(s)\right) d s,
\end{aligned}
$$

for some $v_{*} \in S_{F, x_{*}}$.

Finally, we show there exists an open set $U \subseteq C([0, T], \mathbb{R})$ with $x \notin \Omega_{F}(x)$ for any $\lambda \in(0,1)$ and all $x \in \partial U$. Let $\lambda \in(0,1)$ and $x \in \lambda \Omega_{F}(x)$. Then there exists $v \in L^{1}([0, T], \mathbb{R})$ with $v \in S_{F, x}$ such that, for $t \in[0, T]$, we have

$$
\begin{aligned}
h(t)= & \int_{0}^{t} \frac{(t-s)^{q-1}}{\Gamma(q)} v(s) d s-\lambda_{0} \xi_{1} \int_{0}^{T} \frac{(T-s)^{q-1}}{\Gamma(q)} v(s) d s \\
& +\lambda_{1} \eta_{2} \int_{0}^{T} \frac{(T-s)^{q-2}}{\Gamma(q-1)} v(s) d s+\lambda_{2} \eta_{1} \int_{0}^{T} \frac{(T-s)^{q-3}}{\Gamma(q-2)} v(s) d s \\
& -\mu_{0} \xi_{1} \int_{0}^{T} g_{0}(s, x(s)) d s+\mu_{1} \eta_{2} \int_{0}^{T} g_{1}(s, x(s)) d s \\
& +\mu_{2} \eta_{1} \int_{0}^{T} g_{2}(s, x(s)) d s,
\end{aligned}
$$

and using the computations of the second step above, we have

$$
\begin{aligned}
\|h\| \leq & \psi(\|x\|)\left\{\frac{T^{q-1}}{\Gamma(q)}+\left|\lambda_{0} \xi_{1}\right| \frac{T^{q-1}}{\Gamma(q)}+\left|\lambda_{1} \eta_{2}\right| \frac{T^{q-2}}{\Gamma(q-1)}+\left|\lambda_{2} \eta_{1}\right| \frac{T^{q-3}}{\Gamma(q-2)}\right\} \int_{0}^{T} p(s) d s \\
& +\psi_{0}(\|x\|)\left|\mu_{0} \xi_{1}\right| \int_{0}^{T} p_{0}(s) d s+\psi_{1}(\|x\|)\left|\mu_{1} \eta_{2}\right| \int_{0}^{T} p_{1}(s) d s \\
& +\psi_{2}(\|x\|)\left|\mu_{2} \eta_{1}\right| \int_{0}^{T} p_{2}(s) d s
\end{aligned}
$$




$$
\begin{aligned}
\leq & \psi(\|x\|) \Omega_{1}\|p\|_{L^{1}}+\psi_{0}(\|x\|)\left|\mu_{0} \xi_{1}\right|\left\|p_{0}\right\|_{L^{1}}+\psi_{1}(\|x\|)\left|\mu_{1} \eta_{2}\right|\left\|p_{1}\right\|_{L^{1}} \\
& +\psi_{2}(\|x\|)\left|\mu_{2} \eta_{1}\right|\left\|p_{2}\right\|_{L^{1}} .
\end{aligned}
$$

Consequently, we have

$$
\begin{aligned}
& \|x\| /\left(\psi(\|x\|) \Omega_{1}\|p\|_{L^{1}}+\psi_{0}(\|x\|)\left|\mu_{0} \xi_{1}\right|\left\|p_{0}\right\|_{L^{1}}+\psi_{1}(\|x\|)\left|\mu_{1} \eta_{2}\right|\left\|p_{1}\right\|_{L^{1}}\right. \\
& \left.\quad+\psi_{2}(\|x\|)\left|\mu_{2} \eta_{1}\right|\left\|p_{2}\right\|_{L^{1}}\right) \leq 1 .
\end{aligned}
$$

In view of $\left(\mathrm{H}_{4}\right)$, there exists $M$ such that $\|x\| \neq M$. Let us set

$$
U=\{x \in C([0, T], \mathbb{R}):\|x\|<M\} .
$$

Note that the operator $\Omega_{F}: \bar{U} \rightarrow \mathcal{P}(C([0, T], \mathbb{R}))$ is upper semicontinuous and completely continuous. From the choice of $U$, there is no $x \in \partial U$ such that $x \in \lambda \Omega_{F}(x)$ for some $\lambda \in$ $(0,1)$. Consequently, by the nonlinear alternative of Leray-Schauder type (Lemma 3.1), we deduce that $\Omega_{F}$ has a fixed point $x \in \bar{U}$ which is a solution of problem (1.1). This completes the proof.

\subsection{The lower semicontinuous case}

This section deals with the case when $F$ is not necessarily convex valued. Our strategy to deal with this problem is based on the nonlinear alternative of Leray-Schauder type together with the selection theorem of Bressan and Colombo for lower semi-continuous maps with decomposable values.

Lemma 3.4 (Bressan and Colombo [34]) Let $Y$ be a separable metric space, and let $N$ : $Y \rightarrow \mathcal{P}\left(L^{1}([0, T], \mathbb{R})\right)$ be a multivalued operator satisfying the property $(B C)$. Then $N$ has a continuous selection, that is, there exists a continuous function (single-valued) $g: Y \rightarrow$ $L^{1}([0, T], \mathbb{R})$ such that $g(x) \in N(x)$ for every $x \in Y$.

Theorem 3.5 Assume that $\left(\mathrm{H}_{2}\right),\left(\mathrm{H}_{3}\right),\left(\mathrm{H}_{4}\right)$ and the following condition hold:

$\left(\mathrm{H}_{4}\right) \quad F:[0, T] \times \mathbb{R} \rightarrow \mathcal{P}(\mathbb{R})$ is a nonempty compact-valued multivalued map such that

(a) $(t, x) \mapsto F(t, x)$ is $\mathcal{L} \otimes \mathcal{B}$ measurable;

(b) $x \longmapsto F(t, x)$ is lower semicontinuous for each $t \in[0, T]$;

then the boundary value problem (1.1) has at least one solution on $[0, T]$.

Proof It follows from $\left(\mathrm{H}_{2}\right)$ and $\left(\mathrm{H}_{4}\right)$ that $F$ is of l.s.c. type. Then from Lemma 3.4, there exists a continuous function $f: A C^{1}([0, T], \mathbb{R}) \rightarrow L^{1}([0, T], \mathbb{R})$ such that $f(x) \in \mathcal{F}(x)$ for all $x \in C([0, T], \mathbb{R})$.

Consider the problem

$$
\left\{\begin{array}{l}
{ }^{c} D^{q} x(t) x(t)=f(x(t)), \quad t \in[0, T], \\
x^{(j)}(0)-\lambda_{j} x^{(j)}(T)=\mu_{j} \int_{0}^{T} g_{j}(s, x(s)) d s, \quad j=0,1,2 .
\end{array}\right.
$$

Observe that if $x \in A C^{1}([0, T], \mathbb{R})$ is a solution of (3.1), then $x$ is a solution to problem (1.1). In order to transform problem (3.1) into a fixed point problem, we define the operator 
$\overline{\Omega_{F}}$ as

$$
\begin{aligned}
\overline{\Omega_{F}} x(t)= & \int_{0}^{t} \frac{(t-s)^{q-1}}{\Gamma(q)} f(x(s)) d s-\lambda_{0} \xi_{1} \int_{0}^{T} \frac{(T-s)^{q-1}}{\Gamma(q)} f(x(s)) d s \\
& +\lambda_{1} \eta_{2} \int_{0}^{T} \frac{(T-s)^{q-2}}{\Gamma(q-1)} f(x(s)) d s+\lambda_{2} \eta_{1} \int_{0}^{T} \frac{(T-s)^{q-3}}{\Gamma(q-2)} f(x(s)) d s \\
& -\mu_{0} \xi_{1} \int_{0}^{T} g_{0}(s, x(s)) d s+\mu_{1} \eta_{2} \int_{0}^{T} g_{1}(s, x(s)) d s \\
& +\mu_{2} \eta_{1} \int_{0}^{T} g_{2}(s, x(s)) d s .
\end{aligned}
$$

It can easily be shown that $\overline{\Omega_{F}}$ is continuous and completely continuous. The remaining part of the proof is similar to that of Theorem 3.3. So, we omit it. This completes the proof.

\subsection{The Lipschitz case}

Here we show the existence of solutions for problem (1.1) with a nonconvex valued righthand side by applying a fixed point theorem for multivalued maps due to Covitz and Nadler [35].

Lemma 3.6 ([35]) Let $(X, d)$ be a complete metric space. If $N: X \rightarrow \mathcal{P}_{c l}(X)$ is a contraction, then $\operatorname{Fix} N \neq \emptyset$.

Theorem 3.7 Assume that the following conditions hold:

$\left(\mathrm{A}_{1}\right) F:[0, T] \times \mathbb{R} \rightarrow \mathcal{P}_{c p}(\mathbb{R})$ is such that $F(\cdot, x):[0, T] \rightarrow \mathcal{P}_{c p}(\mathbb{R})$ is measurable for each $x \in \mathbb{R}$;

$\left(\mathrm{A}_{2}\right) H_{d}(F(t, x), F(t, \bar{x})) \leq m(t)|x-\bar{x}|$ for almost all $t \in[0, T]$ and $x, \bar{x} \in \mathbb{R}$ with $m \in$ $L^{1}\left([0, T], \mathbb{R}^{+}\right)$and $d(0, F(t, 0)) \leq m(t)$ for almost all $t \in[0, T] ;$

$\left(\mathrm{A}_{3}\right)$ There exist constants $c_{j}>0, j=0,1,2$, such that

$$
\left|g_{j}(t, x)-g_{j}(t, y)\right| \leq c_{j}|x-y|, \quad \forall t \in[0, T], j=0,1,2, x, y \in \mathbb{R}
$$

Then the boundary value problem (1.1) has at least one solution on $[0, T]$ if

$$
\Omega_{1}\|m\|_{L^{1}}+\left\{c_{0}\left|\mu_{1} \xi_{1}\right|+c_{1}\left|\mu_{2} \eta_{2}\right|+c_{2}\left|\mu_{3} \eta_{1}\right|\right\} T<1
$$

Proof Observe that the set $S_{F, x}$ is nonempty for each $x \in C([0, T], \mathbb{R})$ by the assumption $\left(\mathrm{A}_{1}\right)$, so $F$ has a measurable selection (see Theorem III.6 [36]). Now we show that the operator $\Omega_{F}$, defined in the beginning of the proof of Theorem 3.3, satisfies the assumptions of Lemma 3.6. To show that $\Omega_{F}(x) \in \mathcal{P}_{c l}((C[0, T], \mathbb{R}))$ for each $x \in C([0, T], \mathbb{R})$, let $\left\{u_{n}\right\}_{n \geq 0} \in \Omega_{F}(x)$ be such that $u_{n} \rightarrow u(n \rightarrow \infty)$ in $C([0, T], \mathbb{R})$. Then $u \in C([0, T], \mathbb{R})$ and there exists $v_{n} \in S_{F, x_{n}}$ such that, for each $t \in[0, T]$,

$$
\begin{aligned}
u_{n}(t)= & \int_{0}^{t} \frac{(t-s)^{q-1}}{\Gamma(q)} v_{n}(s) d s-\lambda_{0} \xi_{1} \int_{0}^{T} \frac{(T-s)^{q-1}}{\Gamma(q)} v_{n}(s) d s \\
& +\lambda_{1} \eta_{2} \int_{0}^{T} \frac{(T-s)^{q-2}}{\Gamma(q-1)} v_{n}(s) d s+\lambda_{2} \eta_{1} \int_{0}^{T} \frac{(T-s)^{q-3}}{\Gamma(q-2)} v_{n}(s) d s
\end{aligned}
$$




$$
\begin{aligned}
& -\mu_{0} \xi_{1} \int_{0}^{T} g_{0}(s, x(s)) d s+\mu_{1} \eta_{2} \int_{0}^{T} g_{1}(s, x(s)) d s \\
& +\mu_{2} \eta_{1} \int_{0}^{T} g_{2}(s, x(s)) d s .
\end{aligned}
$$

As $F$ has compact values, we pass onto a subsequence (if necessary) to obtain that $v_{n}$ converges to $v$ in $L^{1}([0, T], \mathbb{R})$. Thus, $v \in S_{F, x}$ and for each $t \in[0, T]$, we have

$$
\begin{aligned}
u_{n}(t) \rightarrow u(t)= & \int_{0}^{t} \frac{(t-s)^{q-1}}{\Gamma(q)} v(s) d s-\lambda_{0} \xi_{1} \int_{0}^{T} \frac{(T-s)^{q-1}}{\Gamma(q)} v(s) d s \\
& +\lambda_{1} \eta_{2} \int_{0}^{T} \frac{(T-s)^{q-2}}{\Gamma(q-1)} v(s) d s+\lambda_{2} \eta_{1} \int_{0}^{T} \frac{(T-s)^{q-3}}{\Gamma(q-2)} v(s) d s \\
& -\mu_{0} \xi_{1} \int_{0}^{T} g_{0}(s, x(s)) d s+\mu_{1} \eta_{2} \int_{0}^{T} g_{1}(s, x(s)) d s \\
& +\mu_{2} \eta_{1} \int_{0}^{T} g_{2}(s, x(s)) d s .
\end{aligned}
$$

Hence, $u \in \Omega(x)$.

Next we show that there exists $\delta<1$ such that

$$
H_{d}\left(\Omega_{F}(x), \Omega_{F}(\bar{x})\right) \leq \delta\|x-\bar{x}\| \quad \text { for each } x, \bar{x} \in A C^{1}([0, T], \mathbb{R}) .
$$

Let $x, \bar{x} \in A C^{1}([0, T], \mathbb{R})$ and $h_{1} \in \Omega_{F}(x)$. Then there exists $v_{1}(t) \in F(t, x(t))$ such that, for each $t \in[0, T]$,

$$
\begin{aligned}
h_{1}(t)= & \int_{0}^{t} \frac{(t-s)^{q-1}}{\Gamma(q)} v_{1}(s) d s-\lambda_{0} \xi_{1} \int_{0}^{T} \frac{(T-s)^{q-1}}{\Gamma(q)} v_{1}(s) d s \\
& +\lambda_{1} \eta_{2} \int_{0}^{T} \frac{(T-s)^{q-2}}{\Gamma(q-1)} v_{1}(s) d s+\lambda_{2} \eta_{1} \int_{0}^{T} \frac{(T-s)^{q-3}}{\Gamma(q-2)} v_{1}(s) d s \\
& -\mu_{0} \xi_{1} \int_{0}^{T} g_{0}(s, x(s)) d s+\mu_{1} \eta_{2} \int_{0}^{T} g_{1}(s, x(s)) d s \\
& +\mu_{2} \eta_{1} \int_{0}^{T} g_{2}(s, x(s)) d s .
\end{aligned}
$$

By $\left(\mathrm{H}_{3}\right)$, we have

$$
H_{d}(F(t, x), F(t, \bar{x})) \leq m(t)|x(t)-\bar{x}(t)|
$$

So, there exists $w \in F(t, \bar{x}(t))$ such that

$$
\left|v_{1}(t)-w\right| \leq m(t)|x(t)-\bar{x}(t)|, \quad t \in[0, T] .
$$

Define $U:[0, T] \rightarrow \mathcal{P}(\mathbb{R})$ by

$$
U(t)=\left\{w \in \mathbb{R}:\left|v_{1}(t)-w\right| \leq m(t)|x(t)-\bar{x}(t)|\right\} .
$$


Since the multivalued operator $U(t) \cap F(t, \bar{x}(t))$ is measurable (Proposition III.4 [36]), there exists a function $v_{2}(t)$ which is a measurable selection for $U$. So, $v_{2}(t) \in F(t, \bar{x}(t))$ and for each $t \in[0, T]$, we have $\left|v_{1}(t)-v_{2}(t)\right| \leq m(t)|x(t)-\bar{x}(t)|$.

For each $t \in[0, T]$, let us define

$$
\begin{aligned}
h_{2}(t)= & \int_{0}^{t} \frac{(t-s)^{q-1}}{\Gamma(q)} v_{2}(s) d s-\lambda_{0} \xi_{1} \int_{0}^{T} \frac{(T-s)^{q-1}}{\Gamma(q)} v_{2}(s) d s \\
& +\lambda_{1} \eta_{2} \int_{0}^{T} \frac{(T-s)^{q-2}}{\Gamma(q-1)} v_{2}(s) d s+\lambda_{2} \eta_{1} \int_{0}^{T} \frac{(T-s)^{q-3}}{\Gamma(q-2)} v_{2}(s) d s \\
& -\mu_{0} \xi_{1} \int_{0}^{T} g_{0}(s, x(s)) d s+\mu_{1} \eta_{2} \int_{0}^{T} g_{1}(s, x(s)) d s \\
& +\mu_{2} \eta_{1} \int_{0}^{T} g_{2}(s, x(s)) d s .
\end{aligned}
$$

Thus,

$$
\begin{aligned}
\mid h_{1}(t) & -h_{2}(t) \mid \\
= & \int_{0}^{t} \frac{(t-s)^{q-1}}{\Gamma(q)}\left|v_{1}(s)-v_{2}(s)\right| d s+\left|\lambda_{0} \xi_{1}\right| \int_{0}^{T} \frac{(T-s)^{q-1}}{\Gamma(q)}\left|v_{1}(s)-v_{2}(s)\right| d s \\
& +\left|\lambda_{1} \eta_{2}\right| \int_{0}^{T} \frac{(T-s)^{q-2}}{\Gamma(q-1)}\left|v_{1}(s)-v_{2}(s)\right| d s+\left|\lambda_{2} \eta_{1}\right| \int_{0}^{T} \frac{(T-s)^{q-3}}{\Gamma(q-2)}\left|v_{1}(s)-v_{2}(s)\right| d s \\
& +\left|\mu_{0} \xi_{1}\right| \int_{0}^{T}\left|g_{0}(s, x(s))-g_{0}(s, \bar{x}(s))\right| d s+\left|\mu_{1} \eta_{2}\right| \int_{0}^{T}\left|g_{1}(s, x(s))-g_{1}(s, \bar{x}(s))\right| d s \\
& +\left|\mu_{2} \eta_{1}\right| \int_{0}^{T}\left|g_{2}(s, x(s))-g_{2}(s, \bar{x}(s))\right| d s \\
\leq & \psi(\|x\|)\left\{\frac{T^{q-1}}{\Gamma(q)}+\left|\lambda_{0} \xi_{1}\right| \frac{T^{q-1}}{\Gamma(q)}+\left|\lambda_{1} \eta_{2}\right| \frac{T^{q-2}}{\Gamma(q-1)}+\left|\lambda_{2} \eta_{1}\right| \frac{T^{q-3}}{\Gamma(q-2)}\right\} \\
& \times\|x-\bar{x}\| \int_{0}^{T} m(s) d s \\
& +c_{0}\left|\mu_{1} \xi_{1}\right| T\|x-\bar{x}\|+c_{1}\left|\mu_{2} \eta_{2}\right| T\|x-\bar{x}\|+c_{2}\left|\mu_{3} \eta_{1}\right| T\|x-\bar{x}\| .
\end{aligned}
$$

Hence,

$$
\left\|h_{1}-h_{2}\right\| \leq\left[\Omega_{1}\|m\|_{L^{1}}+\left\{c_{0}\left|\mu_{1} \xi_{1}\right|+c_{1}\left|\mu_{2} \eta_{2}\right|+c_{2}\left|\mu_{3} \eta_{1}\right|\right\} T\right]\|x-\bar{x}\| .
$$

Analogously, interchanging the roles of $x$ and $\bar{x}$, we obtain

$$
\begin{aligned}
H_{d}\left(\Omega_{F}(x), \Omega_{F}(\bar{x})\right) & \leq \delta\|x-\bar{x}\| \\
& \leq\left[\Omega_{1}\|m\|_{L^{1}}+\left\{c_{0}\left|\mu_{1} \xi_{1}\right|+c_{1}\left|\mu_{2} \eta_{2}\right|+c_{3}\left|\mu_{2} \eta_{1}\right|\right\} T\right]\|x-\bar{x}\| .
\end{aligned}
$$

Since $\Omega_{F}$ is a contraction, it follows by Lemma 3.6 that $\Omega_{F}$ has a fixed point $x$ which is a solution of (1.1). This completes the proof. 
Example 3.8 Consider the following boundary value problem of fractional differential inclusions:

$$
\left\{\begin{array}{l}
{ }^{c} D^{5 / 2} x(t) \in F(t, x(t)), \quad t \in[0,1] \\
x(0)+x(1)=\int_{0}^{1} \frac{x(s)}{3(1+s)^{2}} d s \\
x^{\prime}(0)+x^{\prime}(1)=\frac{1}{2} \int_{0}^{1} \frac{e^{s} x(s)}{3\left(1+e^{s}\right)} d s \\
x^{\prime \prime}(0)+x^{\prime \prime}(1)=\frac{1}{3} \int_{0}^{1} \frac{x(s)}{3\left(1+e^{s}\right)} d s
\end{array}\right.
$$

where $F:[0,1] \times \mathbb{R} \rightarrow \mathcal{P}(\mathbb{R})$ is a multivalued map given by

$$
x \rightarrow F(t, x)=\left[\frac{|x|^{3}}{10\left(|x|^{3}+3\right)}, \frac{|\sin x|}{9(|\sin x|+1)}+\frac{1}{12}\right] .
$$

For $f \in F$, we have

$$
|f| \leq \max \left(\frac{|x|^{3}}{10\left(|x|^{3}+3\right)}, \frac{|\sin x|}{9(|\sin x|+1)}+\frac{1}{12}\right) \leq \frac{7}{36}, \quad x \in \mathbb{R} .
$$

Thus,

$$
\|F(t, x)\|_{\mathcal{P}}:=\sup \{|y|: y \in F(t, x)\} \leq \frac{7}{36}=p(t) \psi(\|x\|), \quad x \in \mathbb{R},
$$

with $p(t)=\frac{1}{3}, \psi(\|x\|)=\frac{7}{12}$. Here

$$
g_{0}(t, x)=\frac{x(t)}{3(1+t)^{2}}, \quad g_{1}(t, x)=\frac{e^{t} x(t)}{3\left(1+e^{t}\right)}, \quad g_{2}(t, x)=\frac{x(t)}{3\left(1+e^{t}\right)}
$$

and $\lambda_{0}=\lambda_{1}=\lambda_{2}=-1, \mu_{0}=1, \mu_{1}=\frac{1}{2}, \mu_{2}=\frac{1}{3}$.

Clearly, $\xi_{1}=-1 / 2, \xi_{2}=1 / 4, \xi_{3}=-1 / 16, \eta_{1}=1 / 16, \eta_{2}=1 / 4,\left|g_{0}(t, x)\right| \leq \frac{1}{3}\|x\|,\left|g_{1}(t, x)\right| \leq$ $\frac{1}{3}\|x\|,\left|g_{2}(t, x)\right| \leq \frac{1}{3}\|x\|$ with $\psi(M)=\frac{7}{12}, \psi_{0}(M)=\psi_{1}(M)=\psi_{2}(M)=M,\|p\|_{L^{1}}=\left\|p_{0}\right\|_{L^{1}}=$ $\left\|p_{1}\right\|_{L^{1}}=\left\|p_{2}\right\|_{L^{1}}=\frac{1}{3}$, and $\Omega_{1}=\frac{85 \sqrt{\pi}}{32}$. In view of the condition

$$
\frac{M}{\psi(M) \Omega_{1}\|p\|_{L^{1}}+\psi_{0}(M)\left|\mu_{0} \xi_{1}\right|\left\|p_{0}\right\|_{L^{1}}+\psi_{1}(M)\left|\mu_{1} \eta_{2}\right|\left\|p_{1}\right\|_{L^{1}}+\psi_{2}(M)\left|\mu_{2} \eta_{1}\right|\left\|p_{2}\right\|_{L^{1}}}>1,
$$

we find that $M>\frac{595 \sqrt{\pi}}{904}$. Thus, all the conditions of Theorem 3.3 are satisfied. So, there exists at least one solution of problem $(3.2)$ on $[0,1]$.

\section{Competing interests}

The authors declare that they have no competing interests.

\section{Authors' contributions}

Each of the authors, BA, SKN and AA, contributed to each part of this work equally and read and approved the final version of the manuscript.

\section{Author details}

'Department of Mathematics, Faculty of Science, King Abdulaziz University, P.O. Box 80203, Jeddah, 21589, Saudi Arabia.

${ }^{2}$ Department of Mathematics, University of Ioannina, Ioannina, 451 10, Greece. 


\section{Acknowledgements}

The authors are grateful to the anonymous referees for their useful comments. The research of B. Ahmad and A. Alsaedi was partially supported by the Deanship of Scientific Research (DSR), King Abdulaziz University, Jeddah, Saudi Arabia.

Received: 4 October 2012 Accepted: 12 March 2013 Published: 10 April 2013

\section{References}

1. Samko, SG, Kilbas, AA, Marichev, OI: Fractional Integrals and Derivatives, Theory and Applications. Gordon \& Breach, Yverdon (1993)

2. Podlubny, I: Fractional Differential Equations. Academic Press, San Diego (1999)

3. Kilbas, AA, Srivastava, HM, Trujillo, Jj: Theory and Applications of Fractional Differential Equations. North-Holland Mathematics Studies, vol. 204. Elsevier, Amsterdam (2006)

4. Lakshmikantham, V, Leela, S, Vasundhara Devi, J: Theory of Fractional Dynamic Systems. Cambridge Academic Publishers, Cambridge (2009)

5. Baleanu, D, Diethelm, K, Scalas, E, Trujillo, JJ: Fractional Calculus: Models and Numerical Methods. Series on Complexity, Nonlinearity and Chaos. World Scientific, Boston (2012)

6. Agarwal, RP, Benchohra, M, Hamani, S: A survey on existence results for boundary value problems of nonlinear fractional differential equations and inclusions. Acta Appl. Math. 109, 973-1033 (2010)

7. Bai, ZB: On positive solutions of a nonlocal fractional boundary value problem. Nonlinear Anal. 72, 916-924 (2010)

8. Balachandran, K, Trujillo, Jj: The nonlocal Cauchy problem for nonlinear fractional integrodifferential equations in Banach spaces. Nonlinear Anal. 72, 4587-4593 (2010)

9. Baleanu, D, Mustafa, OG: On the global existence of solutions to a class of fractional differential equations. Comput. Math. Appl. 59, 1835-1841 (2010)

10. Hernandez, E, O'Regan, D, Balachandran, K: On recent developments in the theory of abstract differential equations with fractional derivatives. Nonlinear Anal. 73(10), 3462-3471 (2010)

11. Wang, Y, Liu, L, Wu, Y: Positive solutions for a nonlocal fractional differential equation. Nonlinear Anal. 74, 3599-3605 (2011)

12. Ford, NJ, Morgado, ML: Fractional boundary value problems: analysis and numerical methods. Fract. Calc. Appl. Anal. $14,554-567(2011)$

13. Ahmad, B, Nieto, JJ: Riemann-Liouville fractional integro-differential equations with fractional nonlocal integral boundary conditions. Bound. Value Probl. 2011, Article ID 36 (2011)

14. Ahmad, B, Nieto, JJ, Alsaedi, A: Existence and uniqueness of solutions for nonlinear fractional differential equations with non-separated type integral boundary conditions. Acta Math. Sci. 31, 2122-2130 (2011)

15. Ahmad, B, Ntouyas, SK: Nonlinear fractional differential equations and inclusions of arbitrary order and multi-strip boundary conditions. Electron. J. Differ. Equ. 2012, Article ID 98 (2012)

16. Wang, G, Agarwal, RP, Cabada, A: Existence results and the monotone iterative technique for systems of nonlinear fractional differential equations. Appl. Math. Lett. 25, 1019-1024 (2012)

17. Bai, ZB, Sun, W: Existence and multiplicity of positive solutions for singular fractional boundary value problems. Comput. Math. Appl. 63, 1369-1381 (2012)

18. Sakthivel, R, Mahmudov, NI, Nieto, JJ: Controllability for a class of fractional-order neutral evolution control systems. Appl. Math. Comput. 218, 10334-10340 (2012)

19. Ahmad, B, Nieto, JJ, Alsaedi, A, El-Shahed, M: A study of nonlinear Langevin equation involving two fractional orders in different intervals. Nonlinear Anal., Real World Appl. 13, 599-606 (2012)

20. Ahmad, B, Ntouyas, SK: Existence results for nonlocal boundary value problems for fractional differential equations and inclusions with strip conditions. Bound. Value Probl. 2012, Article ID 55 (2012)

21. Agarwal, RP, O'Regan, D, Stanek, S: Positive solutions for mixed problems of singular fractional differential equations. Math. Nachr. 285(1), 27-41 (2012)

22. Ahmad, B, Ntouyas, SK: A boundary value problem of fractional differential equations with anti-periodic type integral boundary conditions. J. Comput. Anal. Appl. 15, 1372-1380 (2013)

23. Henderson, J, Ouahab, A: Fractional functional differential inclusions with finite delay. Nonlinear Anal. 70, 2091-2105 (2009)

24. Chang, Y-K, Nieto, JJ: Some new existence results for fractional differential inclusions with boundary conditions. Math. Comput. Model. 49, 605-609 (2009)

25. Cernea, A: On the existence of solutions for nonconvex fractional hyperbolic differential inclusions. Commun. Math. Anal. 9(1), 109-120 (2010)

26. Hamani, S, Benchohra, M, Graef, JR: Existence results for boundary-value problems with nonlinear fractional differential inclusions and integral conditions. Electron. J. Differ. Equ. 2010, Article ID 20 (2010)

27. Agarwal, RP, Ahmad, B, Alsaedi, A, Shahzad, N: On the dimension of the solution set for semilinear fractional differential inclusions. Abstr. Appl. Anal. 2012, Article ID 305924 (2012)

28. Ahmad, B, Ntouyas, SK: Fractional differential inclusions with fractional separated boundary conditions. Fract. Calc. Appl. Anal. 15, 362-382 (2012)

29. Deimling, K: Multivalued Differential Equations. de Gruyter, Berlin (1992)

30. Hu, S, Papageorgiou, N: Handbook of Multivalued Analysis, Theory I. Kluwer Academic, Dordrecht (1997)

31. Kisielewicz, M: Differential Inclusions and Optimal Control. Kluwer Academic, Dordrecht (1991)

32. Granas, A, Dugundji, J: Fixed Point Theory. Springer, New York (2005)

33. Lasota, A, Opial, Z: An application of the Kakutani-Ky Fan theorem in the theory of ordinary differential equations. Bull. Acad. Pol. Sci., Sér. Sci. Math. Astron. Phys. 13, 781-786 (1965)

34. Bressan, A, Colombo, G: Extensions and selections of maps with decomposable values. Stud. Math. 90, 69-86 (1988)

35. Covitz, H, Nadler, SB Jr:: Multivalued contraction mappings in generalized metric spaces. Isr. J. Math. 8, 5-11 (1970)

36. Castaing, C, Valadier, M: Convex Analysis and Measurable Multifunctions. Lecture Notes in Mathematics, vol. 580. Springer, Berlin (1977) 
doi:10.1186/1687-2770-2013-82

Cite this article as: Ahmad et al.: On fractional differential inclusions with anti-periodic type integral boundary conditions. Boundary Value Problems 2013 2013:82.

Submit your manuscript to a SpringerOpen ${ }^{\circ}$ journal and benefit from:

- Convenient online submission

- Rigorous peer review

- Immediate publication on acceptance

Open access: articles freely available online

- High visibility within the field

- Retaining the copyright to your article

Submit your next manuscript at $\boldsymbol{s p r i n g e r o p e n . c o m ~}$ 